\title{
Role of HMGB1 in apoptosis-mediated sepsis lethality
}

\author{
Shixin Qin, ${ }^{1}$ Haichao Wang, ${ }^{2}$ Renqi Yuan, ${ }^{4}$ Hui Li, ${ }^{4}$ Mahendar Ochani, ${ }^{4}$ \\ Kanta Ochani, ${ }^{4}$ Mauricio Rosas-Ballina, ${ }^{4}$ Chris J. Czura, ${ }^{4}$ \\ Jared M. Huston, ${ }^{4}$ Ed Miller, ${ }^{3}$ Xinchun Lin, ${ }^{3}$ Barbara Sherry, ${ }^{5}$ \\ Anjali Kumar, ${ }^{1}$ Greg LaRosa, ${ }^{1}$ Walter Newman, ${ }^{1}$ Kevin J. Tracey, ${ }^{4,5}$ \\ and Huan Yang ${ }^{4,5}$
}

${ }^{1}$ Critical Therapeutics, Inc., Lexington, MA 02421

2Department of Emergency Medicine, ${ }^{3}$ Department of Surgery, North Shore University Hospital, ${ }^{4}$ Laboratory of Biomedical Science, and ${ }^{5}$ Center for Immunology and Inflammation, The Feinstein Institute for Medical Research, Manhasset, NY 11030

Severe sepsis, a lethal syndrome after infection or injury, is the third leading cause of mortality in the United States. The pathogenesis of severe sepsis is characterized by organ damage and accumulation of apoptotic lymphocytes in the spleen, thymus, and other organs. To examine the potential causal relationships of apoptosis to organ damage, we administered Z-VAD-FMK, a broad-spectrum caspase inhibitor, to mice with sepsis. We found that Z-VAD-FMK-treated septic mice had decreased levels of high mobility group box 1 (HMGB1), a critical cytokine mediator of organ damage in severe sepsis, and suppressed apoptosis in the spleen and thymus. In vitro, apoptotic cells activate macrophages to release HMGB1. Monoclonal antibodies against HMGB1 conferred protection against organ damage but did not prevent the accumulation of apoptotic cells in the spleen. Thus, our data indicate that HMGB1 production is downstream of apoptosis on the final common pathway to organ damage in severe sepsis.

\section{CORRESPONDENCE}

Huan Yang:

hyang@nshs.edu
Sepsis is a systemic inflammatory syndrome that can lead to lethal organ damage. Despite advances in modern intensive care, the overall mortality of severe sepsis exceeds $30 \%$, with annual health care costs estimated at $\$ 16.7$ billion in the United States (1). It is the third leading cause of death after cardiovascular disease and cancer. Death from severe sepsis occurs because organs become dysfunctional, especially the lung, liver, and kidney (2-4). Recent studies of the pathophysiology of organ failure in sepsis have implicated apoptosis (5-7). During normal embryonic development and in physiological cell turnover in tissues, apoptotic cells are eliminated by phagocytes via a mechanism that does not activate an inflammatory response. During severe sepsis in animals and humans, however, apoptotic lymphocytes accumulate in the spleen and thymus (5-7). It has been proposed that these apoptotic lymphocytes underlie, at least in part, the pathogenesis of organ damage in severe sepsis. Administration of experimental agents that block apoptosis (e.g., caspase inhibitors) reduces the lethality of severe sepsis in animals $(5,8)$, but the mechanism linking organ failure and death to apoptosis in severe sepsis was previously unknown.

Recent evidence indicates that high mobility group box 1 (HMGB1), a ubiquitous nuclear protein, is a cytokine that mediates organ damage in severe sepsis $(9,10)$. High levels of HMGB1 accumulate in animals and humans with severe sepsis $(9,10)$. Administration of HMGB1 to experimental animals causes lethal organ damage via a mechanism that is dependent on the development of epithelial cell dysfunction $(9,11)$. Passive immunization with neutralizing antiHMGB1 antibodies prevents organ damage and epithelial dysfunction in mice subjected to severe sepsis $(10,11)$. Together, these observations establish HMGB1 as a necessary and sufficient mediator of organ damage in severe sepsis. In this study, we show that apoptotic cells stimulate macrophages to release HMGB1, an event that is proximal in mediating the development of organ damage in severe sepsis.

\section{RESULTS AND DISCUSSION}

We first examined the effects of caspase inhibitors on bacterial endotoxin-induced HMGB1 
A
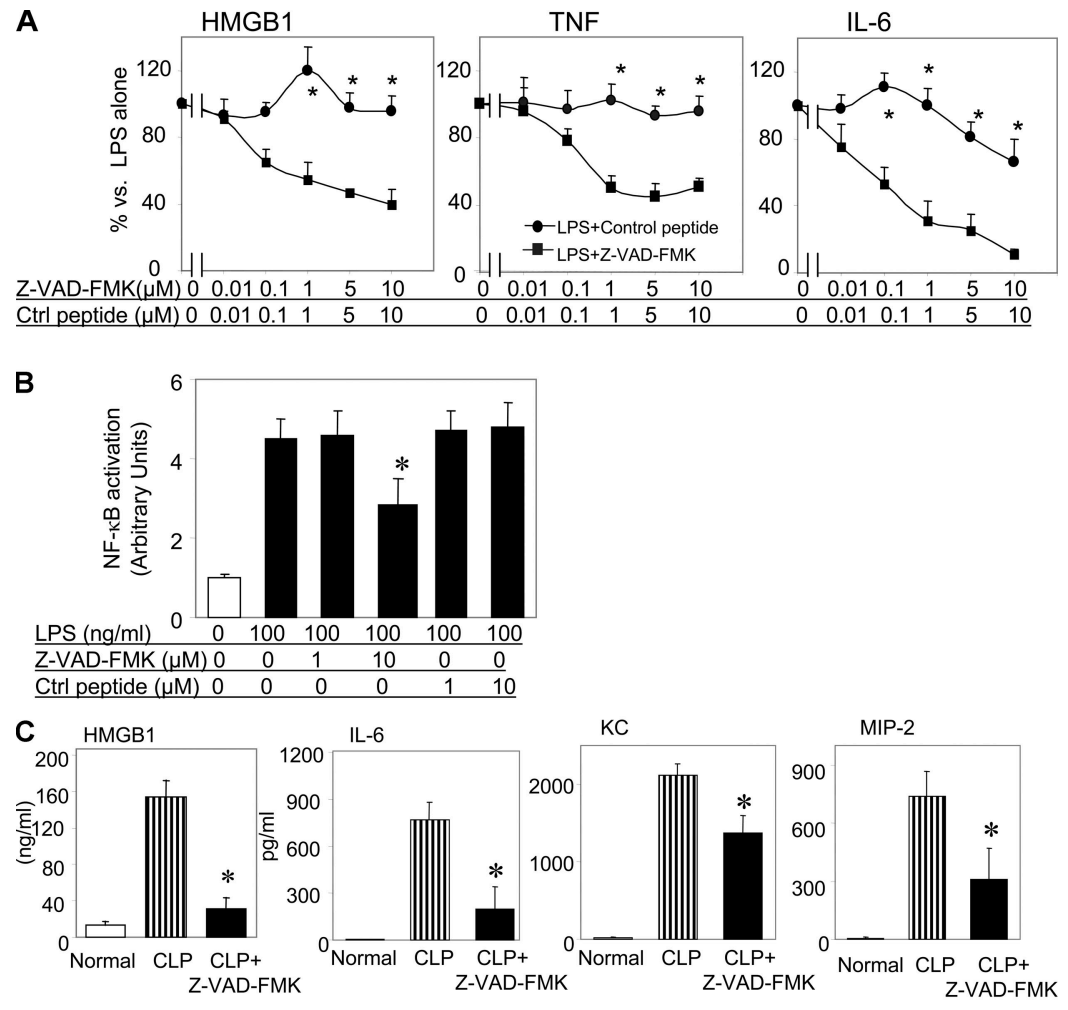

D Normal

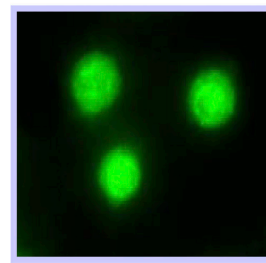

LPS

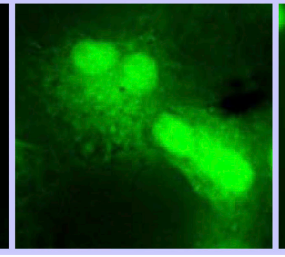

Figure 1. Caspase inhibitor Z-VAD-FMK reduces LPS-induced cytokine release, HMGB1 translocation, and NF- $\kappa$ B activation in RAW 264.7 cells and reduces sepsis-induced serum cytokine levels in mice. (A) Mouse macrophage-like RAW 264.7 cells were stimulated with $200 \mathrm{ng} / \mathrm{ml}$ LPS in the presence of Z-VAD-FMK or control peptide (Z-FA-FMK) at doses indicated for $16 \mathrm{~h}$. Levels of HMGB1, TNF, and IL-6 in conditioned media were measured, and data are expressed as percentages of stimulation by LPS alone. * $P<0.05$ versus LPS + control group; $n=7$. (B) RAW 264.7 cells were stimulated with $100 \mathrm{ng} / \mathrm{ml}$ LPS in the presence of 1 or $10 \mu \mathrm{M}$ Z-VAD-FMK or control peptide for $6 \mathrm{~h}$ at $37^{\circ} \mathrm{C}$. Nuclear extracts were prepared, and EMSA was performed using biotin-labeled nucleotides to measure NF- $\mathrm{KB}$ content. ${ }^{*}, \mathrm{P}<0.05$ versus LPS alone; $n=5-7$ experiments.

release in mouse macrophage-like RAW 264.7 cells. Z-VADFMK (benzyloxycarbonyl-Val-Ala-Asp-fluoromethylketone), a broad-spectrum caspase inhibitor, concentration-dependently inhibited HMGB1 release from LPS-stimulated cultures as compared with the control peptide Z-FA-FMK (benzyloxycarbonyl-Phe-Ala-fluoromethylketone). Similarly, Z-VAD-FMK significantly reduced the LPS-induced release of TNF and IL-6 (Fig. 1 A). Z-VAD-FMK decreased the nuclear translocation of $N F-\kappa B$, which is in agreement with a previously described $\mathrm{NF}-\kappa \mathrm{B}-$ dependent mechanism that
LPS+Z-VAD-FMK LPS+Ctrl peptide

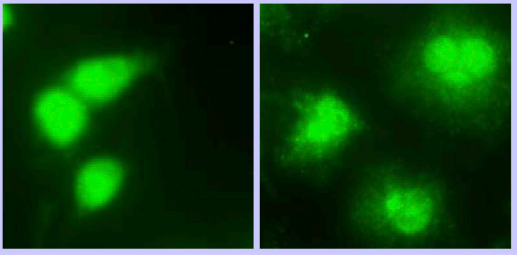

(C) BALB/c mice underwent CLP and received either Z-VAD-FMK or control peptide ( $0.5 \mathrm{mg} /$ mouse) injected intraperitoneally at $90 \mathrm{~min}$ and $12 \mathrm{~h}$ after CLP. Mice were killed $24 \mathrm{~h}$ after CLP surgery. Serum levels of HMGB1, IL-6, $\mathrm{KC}$, and MIP-2 were measured. ${ }^{*}, \mathrm{P}<0.05$ versus CLP group; $n=7-11$ mice per group. Error bars represent SEM. (D) RAW 264.7 cells were incubated with $100 \mathrm{ng} / \mathrm{ml}$ LPS alone or in the presence of $1 \mu \mathrm{m}$ Z-VAD-FMK or control peptide for $16 \mathrm{~h}$. Cells were then incubated with polyclonal antiHMGB1 antibodies followed by FITC-labeled anti-rabbit antibodies and viewed by fluorescent confocal microscopy. Note that LPS caused the export of HMGB1 into the cytosol, whereas Z-VAD-FMK (but not control peptide) prevented its cytoplasmic translocation and preserved HMGB1 in the nucleus. Data are representative of four separate experiments.

regulates LPS-induced HMGB1 release (Fig. $1 \mathrm{~B}$; reference 12). We next subjected mice to severe sepsis in a standardized model of cecal ligation and puncture (CLP; reference 10). Animals received either Z-VAD-FMK or control peptide (Z-FA-FMK). Consistent with a previous study (5), Z-VADFMK substantially prevented the accumulation of apoptotic cells in the spleen and thymus, as measured by nuclear DNA condensation and fragmentation using terminal deoxynucleotidyl transferase-mediated dUTP nick end labeling (TUNEL) assay and caspase 3 staining (unpublished data). 

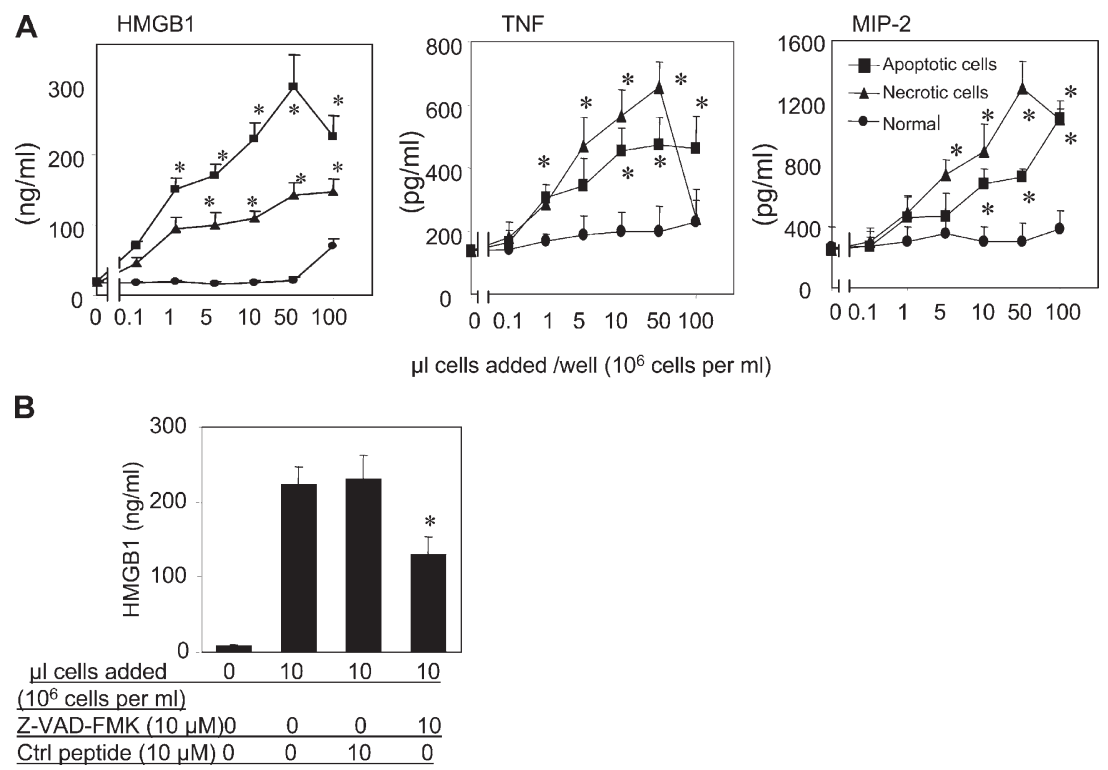

Figure 2. Necrotic and apoptotic cells induce HMGB1, TNF, and MIP-2 release in RAW 264.7 cells; Z-VAD-FMK inhibits apoptotic cell-induced HMGB1 release. (A) Normal, apoptotic, or necrotic cells were added to RAW 264.7 cells at the amounts indicated for $16 \mathrm{~h}$ at $37^{\circ} \mathrm{C}$. HMGB1, TNF, and MIP-2 released in conditioned media were measured. ${ }^{*}, \mathrm{P}<0.05$ versus normal control group; $n=6-8$ independent experiments.

(B) Apoptotic cells were added to RAW 264.7 cells at the amounts indicated in the presence of $10 \mu \mathrm{M}$ Z-VAD-FMK or control peptide for $16 \mathrm{~h}$ at $37^{\circ} \mathrm{C}$, and the HMGB1 released was measured by Western blotting $(9,10) .{ }^{*}, P<0.05$ versus apoptotic cells alone; $n=6-8$ independent experiments. Error bars represent SEM.
Furthermore, it significantly attenuated the sepsis-induced systemic accumulation of HMGB1, IL-6, keratinocyte-derived chemokine $(\mathrm{KC})$, and macrophage inflammatory protein 2 (MIP-2; Fig. 1 C). Z-VAD-FMK effectively blocked the translocation of HMGB1 from the cellular nucleus to the cytoplasm (Fig. 1 D). Collectively, these data indicate that Z-VAD-FMK attenuates HMGB1 release by decreasing the NF-кB-dependent translocation of HMGB1 from the nucleus to the cytosol.

Exposure of apoptotic cells to macrophage cultures stimulates the release of HMGB1 in a dose-dependent manner. Similarly, exposure of macrophages to apoptotic cells also induced a small but significant release of TNF and MIP-2 from macrophages (Fig. 2 A). HMGB1 release was observed after the exposure of macrophages to both apoptotic T cells (unpublished data) and apoptotic macrophages. In agreement with a previous study (12), necrotic cell debris also stimulated HMGB1 release (Fig. 2 A). Z-VAD-FMK inhibited apoptotic cell-induced HMGB1 release (Fig. 2 B), indicating that the accumulation of apoptotic cells can activate macrophages to release HMGB1 and other proinflammatory cytokines.

To determine whether inhibiting HMGB1 attenuates the accumulation of apoptotic cells, we administered neutralizing anti-HMGB1 mAbs (Fig. 3 A) to mice with severe sepsis. Dose-response experiments revealed that treatment with anti-HMGB1 $\mathrm{mAb}$ significantly reduced sepsis lethality (Fig. 3 A). As shown in Fig. 3 B, serum levels of lactate dehydrogenase $(\mathrm{LDH})$, blood urea nitrogen (BUN), alkaline phosphatase, serum glutamate pyruvate transaminase (SGPT), and serum glutamate oxaloacetate transaminase (SGOT) were all elevated in severe sepsis and significantly reduced by treatment with anti-HMGB1 mAb as compared with controls receiving nonimmune $\mathrm{IgG}$. Spleen bacterial counts were not significantly different between treatment groups (unpublished data). Anti-HMGB1 mAb also significantly reduced serum levels of proinflammatory cytokines and chemokines, including IL-6, KC, and MIP- $1 \alpha$ but not the anti-inflammatory cytokine IL-10 (Fig. 3 C). Together, these experimental data suggest that anti-HMGB1 mAb confers protection against severe sepsis by preventing the development of organ damage.

TUNEL assay, caspase 3, and Annexin VI staining revealed that apoptotic cells accumulated in the spleen of mice with severe sepsis (Fig. 4 A, top). No apparent apoptosis was observed in the kidney of these mice (unpublished data). Even though the administration of anti-HMGB1 mAb did not alter apoptosis in the spleen, HMGB1 mAb reduced sepsis-induced focal apoptosis in the liver (Fig. 4 A, bottom). Pathological evaluation revealed sepsis-induced lung injury with alveolar septal thickening, cellular infiltrates, alveolar congestion, hemorrhage, and edema in septic mice. Treatment with anti-HMGB1 mAb substantially decreased the severity of acute lung injury (Fig. 4 B). Sepsis-induced liver injury (focal necrosis and inflammation) was also reduced by the administration of anti-HMGB1 mAb (Fig. 4 B). No apparent differences were observed in tissue sections obtained from anti-HMGB1 $\mathrm{mAb}$ and $\operatorname{IgG}$ treatment groups in the kidney (Fig. 4 B), heart, and small intestine (not depicted). 
A

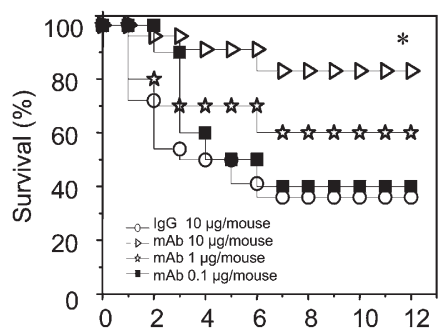

B
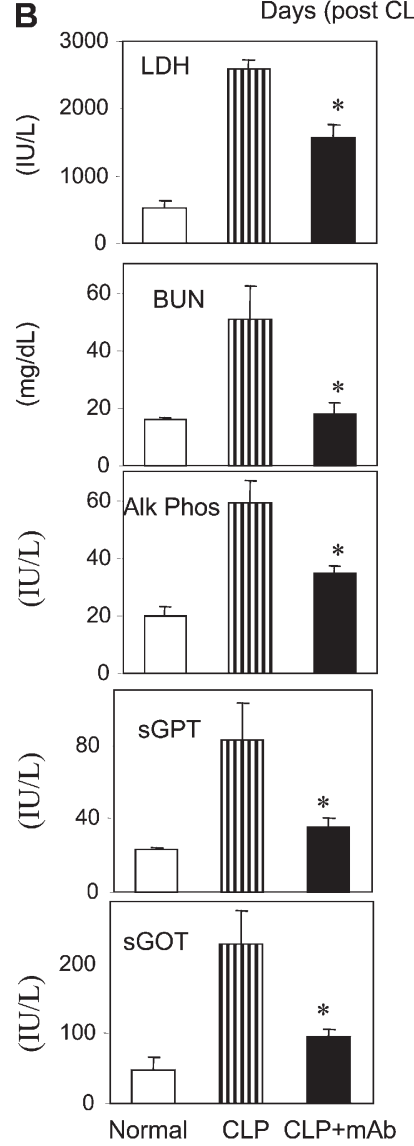

C
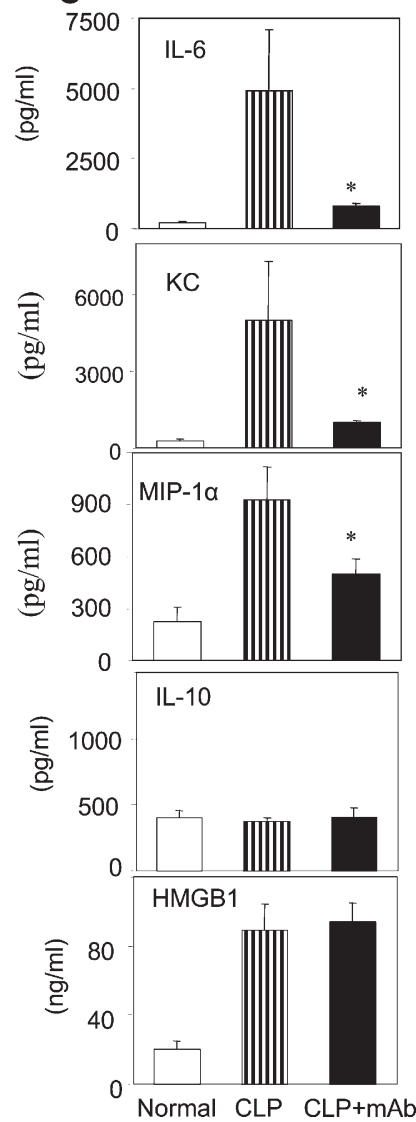

Figure 3. Treatment with anti-HMGB1 mAb increases survival and reduces serum levels of cytokines and enzymes in sepsis induced by CLP. (A) BALB/c mice were subjected to CLP surgery $(n=10-22$ mice per group). At $24 \mathrm{~h}$ after CLP surgery, mice received a single dose of antiHMGB1 mAb at 0.1, 1, or $10 \mu \mathrm{g} /$ mouse or control lgG injected intraperitoneally. No significant difference was observed in mice treated with different amounts of $\lg G(0.1,1$, or $10 \mu \mathrm{g} /$ mouse; not depicted). Animal survival was monitored for 2 wk. ${ }^{*}, P<0.01$ versus the lgG-treated group. (B and $C$ ) $\mathrm{BALB} / \mathrm{c}$ mice had CLP surgery and received anti-HMGB1 mAb or nonimmune $\lg \mathrm{G}$ administered intraperitoneally at $10 \mu \mathrm{g}$ per mouse $24 \mathrm{~h}$ after CLP and were killed $40 \mathrm{~h}$ after surgery. (B) Serum levels of LDH, BUN, alkaline phosphatase (Alk Phos), SGPT, and SGOT were measured using commercially obtained assay kits. (C) Serum levels of IL-6, KC, MIP- $1 \alpha$, and IL-10 were measured by ELISAs, and HMGB1 was measured. Data are means \pm SEM (error bars) of 5-13 animals in each group. ${ }^{*}, \mathrm{P}<0.05$ versus CLP group.
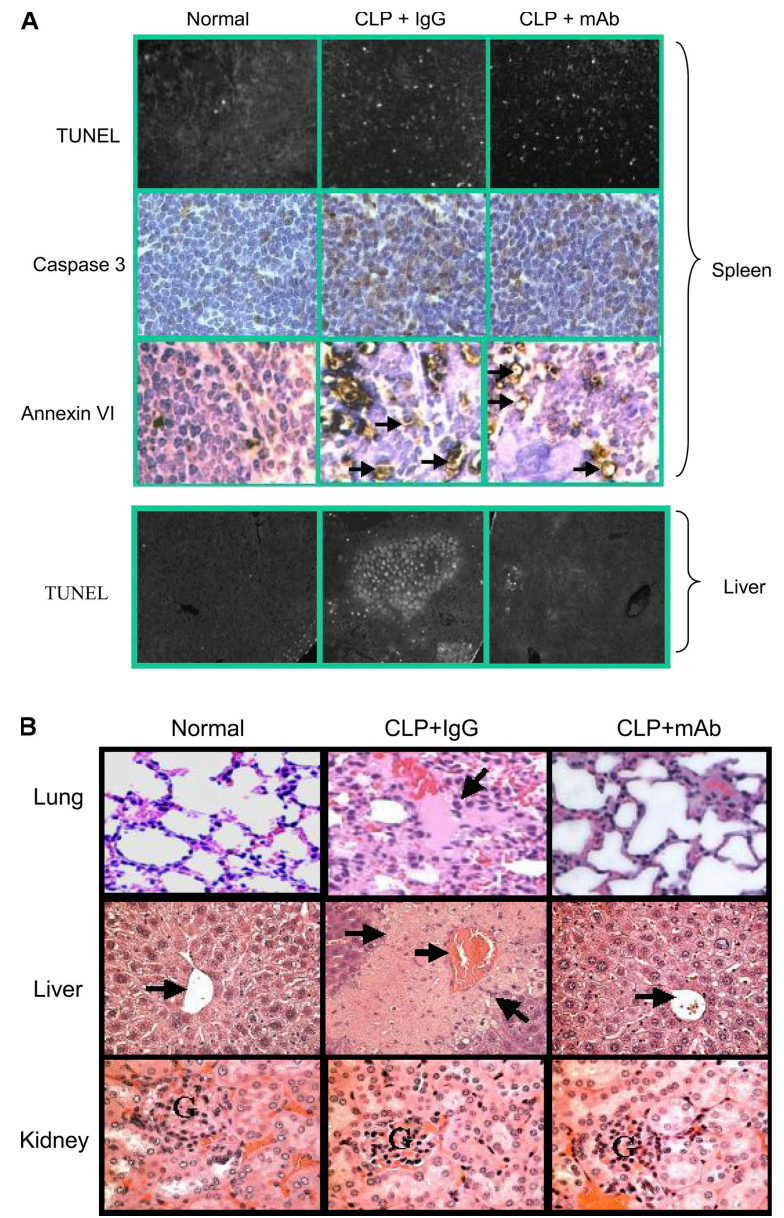

Figure 4. Anti-HMGB1 mAb reduces serum levels of proinflammatory cytokines and organ damage without altering apoptosis in the spleen of septic mice. BALB/c mice were subjected to CLP surgery and received anti-HMGB1 $\mathrm{mAb}$ or nonimmune $\lg \mathrm{G}$ administered intraperitoneally at $10 \mu \mathrm{g} /$ mouse $24 \mathrm{~h}$ after CLP. Mice were killed $40 \mathrm{~h}$ after CLP surgery. In some experiments, between 30 and $72 \mathrm{~h}$ after CLP, any mice that looked very ill were killed (another mouse was killed from the control group at the same time). (A) Tissue sections of spleens (top) and livers (bottom) of normal or septic mice were prepared by using the standard formalin-fixed, paraffin-embedded procedure and were mounted on glass slides, and TUNEL assay, caspase 3 (top), and Annexin VI (top) stainings were performed. Arrows indicate the positive-staining cells. (B) Tissues were stained with hematoxylin and eosin. (top left) Normal lung shows thin alveolar septal wall and normal cellularity. (top middle) Lung from a septic mouse showing alveolar septal wall thickening, increase in cellular infiltrates, alveolar congestion, hemorrhage, and edema (arrow). (top right) Lung from a septic mouse treated with anti-HMGB1 mAb showing near normal alveoli with thin septum. (middle left) Normal liver showing a central vein (arrow) surrounded by hepatocytes and sinusoids. (middle) Liver from a septic mouse showing a congested central vein (arrow) and necrotic lesions (arrows) as revealed by the loss of cells and the structure of hepatic acinus. (middle right) Liver from a septic mouse treated with anti-HMGB1 mAb showing a central vein (arrow) and surrounding near normal hepatocytes. (bottom left) Normal kidney showing cortex with the glomerulus (G). (bottom middle) Kidney from a septic mouse showing the cortex with swelling tubule epithelia and congestion. (bottom right) Kidney from a septic mouse treated with anti-HMGB1 mAb showing swelling tubule epithelia and congestion. Data are representative of four to eight animals per group. 


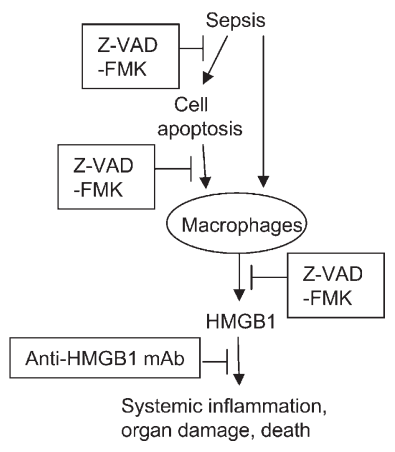

Figure 5. Model of the protective effects of anti-HMGB1 treatment in sepsis lethality. The excessive release of HMGB1 is toxic, and it causes organ damage and death. Sepsis can induce cell apoptosis. Apoptotic cells phagocytosed by macrophages release HMGB1. Caspase inhibitor Z-VAD-FMK inhibits both cell apoptosis and HMGB1 release directly and, thus, improves sepsis survival. Anti-HMGB1 mAbs improve sepsis survival by neutralizing HMGB1 toxicity without altering sepsis-induced apoptosis in the spleen.

Collectively, our data suggest that anti-HMGB1 mAb improves survival in severe sepsis by preventing vital organ damage in lungs and livers without altering the development of sepsis-induced apoptosis in the spleen.

These results provide evidence that administration of the caspase inhibitor Z-VAD-FMK considerably decreases HMGB1 release from macrophages and decreases serum HMGB1 levels in mice with severe sepsis. The relationships between HMGB1 and apoptosis are complex. A previous study demonstrated that HMGB1 can be passively released by necrotic cells, thereby transmitting the "injury" signal to neighboring immune cells (12). However, HMGB1 is not released by apoptotic cells, which dissolve without setting off an inflammatory response. Our experimental data indicate that exposure of macrophages to apoptotic cells activates HMGB1 release. Accordingly, it appears that the excessive accumulation of apoptotic cells in severe sepsis can activate a downstream release of HMGB1, which mediates organ damage (Fig. 5).

Other work has indicated that HMGB1 acts as an inhibitor of apoptosis and as a pro-oncogene in tumorigenesis (13-15). Increased HMGB1 levels are observed in colon tumors and are associated with enhanced levels of antiapoptotic protein c-IAP2 (13). High levels of HMGB1 are also observed in human primary breast carcinoma and in experimental sepsis, endotoxemia, arthritis, and hepatic ischemia reperfusion injury (16-20). HMGB1 levels are increased in human sepsis (9). Previous studies showed that HMGB1 can be passively released by necrotic or damaged cells and actively secreted by macrophages/monocytes and dendritic cells upon stimulation $(9,12,21)$. We have now discovered that apoptotic cells can stimulate HMGB1 release by macrophages in animals with severe sepsis. HMGB1 is downstream of apoptosis on the final common pathway to organ damage.
We previously showed that polyclonal anti-HMGB1 antibodies protect against sepsis lethality (10). In this study, we used HMGB1-specific monoclonal antibodies in a single dose of $10 \mu \mathrm{g}$ per mouse $(0.4 \mathrm{mg} / \mathrm{kg})$ to increase survival in sepsis, indicating a previously unrecognized potency and specificity of anti-HMGB1 treatment in sepsis. Although it had been generally accepted that the clearance of apoptotic cells is via a noninflammatory pathway, it now appears that the accumulation of apoptotic cells may provoke other cells to release HMGB1, which, in turn, mediates severe organ damage.

\section{MATERIALS AND METHODS}

Reagents. 10\% neutral formalin solution, FITC-labeled anti-rabbit IgG, LPS (Escherichia coli; 0111:B4), and camptothecin were obtained from SigmaAldrich. Polycaspase inhibitor Z-VAD-FMK and control peptide Z-FA-FMK were obtained from BD Biosciences. Anticaspase 3 antibodies were purchased from R\&D Systems. Recombinant rat HMGB1 was isolated, and contaminating LPS was removed as previously described $(10,22)$. LPS content is $<0.1 \mathrm{ng} / \mu \mathrm{g}$ of protein $(22)$

Generation of neutralizing mAbs to HMGB1 and epitope mapping. Female BALB/c mice (5-6 wk old) were immunized with recombinant HMGB1 mixed with Freund's adjuvant injected intraperitoneally, and a final boost was given intravenously. Mice were killed after the final boost, and spleen cells were fused with myeloma cells according to a standard protocol. For epitope mapping, overlapping 18-amino acid peptides covering the entire HMGB1 sequence were synthesized by BioSource International. Neutralizing activity of anti-HMGB1 $\mathrm{mAbs}$ was assessed in macrophage cultures exposed to HMGB1 and assayed for the ability to inhibit TNF release (10). Clone 1 was found to bind a region between amino acids 53 and 63, and clone 2 bound to amino acids $67-78$ of the protein, both in the A box region of HMGB1. Both clones were tested in the animal experiments.

Cell culture. Mouse macrophage-like RAW 264.7 cells (American Type Culture Collection) were cultured as previously described (10). Apoptosis was induced by treating the cells with $6 \mu \mathrm{M}$ camptothecin at $37^{\circ} \mathrm{C}$ for $4 \mathrm{~h}$. Necrosis was induced by three cycles of freezing and thawing. Cells were confirmed to be apoptotic by caspase 3 staining. Necrosis was verified by microscopic evaluation showing cell fragments but no intact cells.

EMSA for NF-кB activation. RAW 264.7 cells were stimulated with $100 \mathrm{ng} / \mathrm{ml}$ LPS alone or in the presence of 1 or $10 \mu \mathrm{M}$ Z-VAD-FMK or control peptide Z-FA-FMK for $6 \mathrm{~h}$ at $37^{\circ} \mathrm{C}$. Nuclear extracts were prepared, and electrophoretic mobility shift assay (EMSA) was performed using biotinlabeled oligonucleotides to measure NF- $\mathrm{kB}$ content (Pierce Chemical Co.).

HMGB1 staining and confocal microscopy. RAW 264.7 cells cultured in slide chambers were stimulated with $100 \mathrm{ng} / \mathrm{ml}$ LPS alone or in the presence of $1 \mu \mathrm{M} \mathrm{Z-VAD-FMK}$ or control peptide for $16 \mathrm{~h}$ at $37^{\circ} \mathrm{C}$. Intracellular localization of HMGB1 was determined by using $2.5 \mu \mathrm{g} / \mathrm{ml} \mathrm{HMGB} 1$ antibodies followed by incubation with FITC-labeled anti-rabbit IgG. Cells were mounted for viewing by fluorescent confocal microscopy.

Animal experiments. Male 6-8-wk-old BALB/c mice (Sprague-Dawley; Harlan) were housed in The Feinstein Institute for Medical Research (FIMR) Animal Facility. All animal procedures were approved by the institute's Institutional Animal Care and Use Committee. Mice were subjected to CLP procedure $(10,23)$, and anti-HMGB1 $\mathrm{mAb}$ was given intraperitoneally at $24 \mathrm{~h}$ after CLP. In parallel experiments, mice were killed at various time points to collect blood and tissues (lungs, livers, kidneys, hearts, small intestine, and spleens). Splenic bacteria were recovered as previously described (10). In some experiments, mice were given caspase inhibitors 
(Z-VAD-FMK) or control peptide (Z-FA-FMK; $0.5 \mathrm{mg} / \mathrm{mouse}$ injected intraperitoneally) at $90 \mathrm{~min}$ and $12 \mathrm{~h}$ and were killed $24 \mathrm{~h}$ after CLP.

Measurements of cytokine and serum enzymes. Serum levels of cytokines were determined by ELISA kits (R\&D Systems). Serum levels of BUN, LDH, alkaline phosphatase, SGPT, and SGOT were measured by commercially obtained assay kits. HMGB1 was measured by Western immunoblotting $(9,10)$.

Analyses of tissue histology and apoptosis. Immediately after killing, tissues were fixed, and sections were made and stained with hematoxylin and eosin for morphologic evaluations. For apoptosis analyses, TUNEL staining was performed by using the ApoAlert DNA fragmentation assay kit (BD Biosciences), and caspase 3 expression was determined by using an immunostaining kit (ABC kit; Vector Laboratories).

Statistical analysis. Data are presented as means \pm SEM. Differences between treatment groups were determined by Student's $t$ test and one-way analysis of variance followed by the least significant difference test or Fisher's exact test. $\mathrm{P}<0.05$ was considered statistically significant.

This work was supported, in part, by grants from the FIMR General Clinical Research Center (M01RR018535) and from The National Institute of General Medical Science (to K.J. Tracey).

The authors declare competing financial interests: K.J. Tracey and $\mathrm{H}$. Yang are consultants to Critical Therapeutics, Inc., which has sponsored research and license agreements with FIMR for patents related to inhibiting HMGB1.

There are no other conflicts of interest.

Submitted: 1 November 2005

Accepted: 19 May 2006

\section{REFERENCES}

1. Angus, D., and R.S. Wax. 2001. Epidemiology of sepsis: an update. Crit. Care Med. 29:S109-S116.

2. Riedemann, N.C., R.-F. Guo, and P.A. Ward. 2003. Novel strategies for the treatment of sepsis. Nat. Med. 9:517-524.

3. Tracey, K.J. 2005. Fatal Sequence: the Killer Within. Dana Press, Washington, D.C. 224 pp.

4. Lotze, M.T., and K.J. Tracey. 2005. High-mobility group box 1 protein (HMGB1): nuclear weapon in the immune arsenal. Nat. Rev. Immunol. 5:331-342.

5. Hotchkiss, R., K.C. Chang, P.E. Swanson, K.W. Tinsley, J.J. Hui, P. Klender, S. Xanthoudakis, S. Roy, C. Black, E. Grimm, et al. 2000. Caspase inhibitors improve survival in sepsis: a critical role of the lymphocyte. Nat. Immunol. 1:496-501.

6. Hotchkiss, R.S., and I.E. Karl. 2003. The pathophysiology and treatment of sepsis. N. Engl. J. Med. 348:138-150.

7. Wesche, D.E., J.L. Lomas-Neira, M. Perl, C.S. Chung, and A. Ayala. 2005. Leukocyte apoptosis and its significance in sepsis and shock. J. Leukoc. Biol. 78:325-337.

8. Van Molle, W., G. Denecker, I. Rodriguez, P. Brouckaert, P. Vandenabeele, and C. Libert. 1999. Activation of caspases in lethal experimental hepatitis and prevention by acute phase proteins. J. Immunol. 163:5235-5241.
9. Wang, H., O. Bloom, M. Zhang, J.M. Vishnubhakat, M. Ombrellino, J. Che, A. Frazier, H. Yang, S. Ivanova, L. Borovikova, et al. 1999. HMG-1 as a late mediator of endotoxin lethality in mice. Science. 285:248-251.

10. Yang, H., M. Ochani, J. Li, X. Qiang, M. Tanovic, H.E. Harris, S.M. Susarla, L. Ulloa, H.C. Wang, R. DiRaimo, et al. 2004. Reversing established sepsis with antagonists of endogenous HMGB1. Proc. Natl. Acad. Sci. USA. 101:296-301.

11. Sappington, P.L., R. Yang, H. Yang, K.J. Tracey, R.L. Delude, and M.P. Fink. 2002. HMGB1 B box increases the permeability of Caco-2 enterocytic monolayers and impairs intestinal barrier function in mice. Gastroenterology. 123:790-802.

12. Scaffidi, P., T. Misteli, and M.E. Bianchi. 2002. Release of chromatin protein HMGB1 by necrotic cells triggers inflammation. Nature. 418:191-195.

13. Volp, K., M.L. Brezniceanu, S. Bosser, T. Brabletz, T. Kirchner, D. Gottel, S. Joos, and M. Zornig. 2006. Increased expression of high mobility group box 1 (HMGB1) is associated with an elevated level of the anti-apoptotic c-IAP2 protein in human colon carcinomas. Gut. 55:234-242.

14. Zeh, H.J., III, and M.T. Lotze. 2005. Addicted to death: invasive cancer and the immune response to unscheduled cell death. J. Immunother. 28:1-9.

15. Riuzzi, F., G. Sorci, and R. Donato. 2006. The amphoterin (HMGB1)/ receptor for advanced glycation end products (RAGE) pair modulates myoblast proliferation, apoptosis, adhesiveness, migration, and invasiveness. Functional inactivation of RAGE in L6 myoblasts results in tumor formation in vivo. J. Biol. Chem. 281:8242-8253.

16. Tsung, A., R. Sahai, H. Tanaka, A. Nakao, M.P. Fink, M.T. Lotze, H. Yang, J. Li, K.J. Tracey, D.A. Geller, and T.R. Billiar. 2005. The nuclear factor HMGB1 mediates hepatic injury after murine liver ischemia-reperfusion. J. Exp. Med. 201:1135-1143.

17. Taniguchi, N., K. Kawahara, K. Yone, M. Hashiguchi, and M. Goto. 2003. High mobility group box chromosomal protein 1 plays a role in the pathogenesis of rheumatoid arthritis as a novel cytokine. Arthritis Rheum. 48:971-981.

18. Kokkola, R., E. Sundberg, A.K. Ulfgren, K. Palmblad, J. Li, H. Wang, L. Ulloa, H. Yang, X.J. Yan, R. Furie, et al. 2002. High mobility group box chromosomal protein 1: a novel proinflammatory mediator in synovitis. Arthritis Rheum. 46:2598-2603.

19. Yang, H., H. Wang, C.J. Czura, and K.J. Tracey. 2005. The cytokine activity of HMGB1. J. Leukoc. Biol. 78:1-8.

20. Brezniceanu, M.L., K. Volp, S. Bosser, C. Solbach, P. Lichter, S. Joos, and M. Zornig. 2003. HMGB1 inhibits cell death in yeast and mammalian cells and is abundantly expressed in human breast carcinoma. FASEB J. 17:1295-1297.

21. Czura, C.J., H. Yang, C.A. Amella, and K.J. Tracey. 2004. HMGB1 in the immunology of sepsis (not septic shock) and arthritis. Adv. Immunol. 84:181-200.

22. Li, J., H. Wang, J.M. Mason, J. Levine, M. Yu, L. Ulloa, C.J. Czura, K.J. Tracey, and H. Yang. 2004. Recombinant HMGB1 with cytokinestimulating activity. J. Immunol. Methods. 289:211-223.

23. Wichmann, M.W., J.M. Haisken, A. Ayala, and H. Chaudry. 1996. Melatonin administration following hemorrhagic shock decreases mortality from subsequent septic challenge. J. Surg. Res. 65:109-114. 